\title{
Mounting and Alignment of IXO Mirror Segments
}

\author{
Kai-Wing Chan*a,b, William Zhang ${ }^{\mathrm{b}}$, Tyler Evans ${ }^{\mathrm{b}, \mathrm{c}}$, Ryan McClelland ${ }^{\mathrm{b}, \mathrm{c}}$, Melinda Hong ${ }^{\mathrm{b}, \mathrm{c}}$, James \\ Mazzarella $^{\mathrm{b}, \mathrm{c}}$, Timo Saha ${ }^{\mathrm{b}}$, , Lalit Jalota ${ }^{\mathrm{a}, \mathrm{b}}$, Lawrence Olsen ${ }^{\mathrm{b}}$, Glenn Byron ${ }^{\mathrm{b}, \mathrm{c}}$, \\ ${ }^{a}$ Center for Research and Exploration in Space Science and Technology \& Center for Space Science \\ and Technology, University of Maryland, Baltimore County, Baltimore MD 21250, USA \\ bASA/Goddard Space Flight Center, Greenbelt, MD 20771, USA \\ 'SGT, Inc., 7701 Greenbelt Road, Greenbelt, MD 20770, USA
}

\begin{abstract}
A suspension-mounting scheme is developed for the IXO (International X-ray Observatory) mirror segments in which the figure of the mirror segment is preserved in each stage of mounting. The mirror, first fixed on a thermally compatible strongback, is subsequently transported, aligned and transferred onto its mirror housing. In this paper, we shall outline the requirement, approaches, and recent progress of the suspension mount processes.
\end{abstract}

Keywords: X-ray optics, International X-ray Observatory, IXO telescope, mirror mounting

\section{INTRODUCTION}

An important emphasis of the International X-ray Observatory (IXO) mission is high-resolution imagining spectroscopy. To achieve this goal, a large $\mathrm{x}$-ray grazing telescope is combined with a high energy resolution detector to achieve a large effective area of about $3 \mathrm{~m}^{2}$ in the soft x-ray band $<10 \mathrm{keV}$. A grating system is also use for high-resolution spectroscopy at the lower end of the energy band. ${ }^{1}$ Segmented, grazing incidence, Wolter type I optics, is chosen to provide the large effective area. The mission requirement for angular resolution is 5 arc-seconds, measured in halfpower-diameter (HPD). This is a significant technological challenge to meet, considering the required compactness of the telescope's reflecting surfaces. The challenges include forming the mirror into precise optical forms, aligning the mirror, and mounting them. Metrology of the mirrors, sub-systems and the full telescope all needs technology development. In this paper, we will focus on the effort of mirror mounting, at NASA's Goddard Space Flight Center, to meet the challenges posed by the thinness of the mirror segments.

To meet the mission requirements, a baseline telescope design was developed at NASA in which the optical system, known as the Flight Mirror Assembly (FMA) is $3.3 \mathrm{~m}$ in diameter, with a focal length of $20 \mathrm{~m}$. The design of the telescope's construction is modular, ${ }^{2,3}$. It consists of a nest of 361 concentric shells, distributed in 3 rings. Each shell consists of segmented pairs of mirrors, integrated into separate modules. There are all together 60 modules: 12 inner, 24 middle and 24 outer modules. Each module consists of from 100 to 140 mirror shells (and twice that many mirror segments). The radial breaks of the sections are chosen for optimal optical throughput and also from the consideration that the mirror segments, nominally $15^{\circ}$ or $30^{\circ}$ in angular span, are not wider than $400 \mathrm{~mm}$ in azimuthal length. The axial length of the mirror segment is $200 \mathrm{~mm}$ for each stage of primary and secondary x-ray reflection. The mirrors are formed from $0.4 \mathrm{~mm}$ thick glass substrates.

The current error budget calls for an FMA-level requirement of angular resolution of 4 arc-seconds. Flow-down of the error budget allocates 2.4 arc-seconds for individually mounted mirror. The crucial technologies that are needed to be developed to meet these requirements, specifically, are: first, to develop a glass forming technique that allows fabricating mirror at the 2 arc-seconds level with glass of thickness of $0.4 \mathrm{~mm}$; and secondly, to develop a mirror handling technique which allows aligning and mounting these lightweight mirror segments with sub-arc-second mounting error. These mirror segments can be thermally formed on precise mandrel. The forming process ("slumping") aims for the glass segment to take up precise low order figure. High-frequency surface roughness, which is inherent in the glass and is acceptable in the original smooth glass substrate, is preserved in the thermoforming. ${ }^{4}$ The mounting processes, which 
include mounting of individual mirrors for measurement (the metrology mount) as well as the process that allows the mirror to be aligned and integrated, are the subject of this paper.

\subsection{Challenges and constrains}

Currently, at NASA's Goddard Space Flight Center, the baseline design of the FMA consists of over 14,000 segments, each of $0.4 \mathrm{~mm}$ thick. (An entirely different approach, not addressed here, is pursued by ESA where a module is formed by stacking specially wedged silicon wafer. ${ }^{5}$ ) The mirror surface area and mass permitted by the spacecraft require an areal density of the mirror segments of roughly $1 \mathrm{~kg} / \mathrm{m}^{2}$. With the choice of glass substrate (with a mass density of $\sim 2.5$ $\left.x 10^{3} \mathrm{~kg} / \mathrm{m}^{3}\right)$, the thickness of these mirrors is therefore limited to approximately $0.4 \mathrm{~mm}$. Substrate substantially thicker than this will not be able to meet the mass or the area requirement. Within these broad parameters, the critical challenge in the optics thus lies in attaining the angular resolution with these thin glass mirrors, and to produce, align and mount them within schedule. Given this axial length of $200 \mathrm{~mm}, 1 \mu \mathrm{m}$ error peak-to-valley in low order axial figure error, for example, in sag, results in an error of about 8 arc-seconds in the mirror's axial performance, measured in HPD. The mounting, which produces figure distortion of low order, therefore requires sub- $\mu \mathrm{m}$ precision. The limit on the amplitude of figure distortion is even more stringent for error of higher spatial frequency, which may occur depending on the number of boundary points in mounting the mirrors.

\section{MIRROR MOUNTING}

Besides figure imperfection in the mirror to be mounted (which may come from error in forming these mirrors, or from distortion due to coating of glass segments), sources of mirror distortion include: (1) gravity sag during metrology and other ground tests, during assembly, and gravity relief after launch, (2) over-constraint of the mirrors from mounting, and (3) thermal distortion. For the purpose of the present discussion on mirror mounting, we will not address the figure error in the original mirror segments, such as errors when forming the mirrors and from coating them. The mirrors, depending on how they are affixed into the telescope housing and the mirror assembly's thermal control, will suffer from strain arising from CTE (coefficient of thermal expansion) mismatch, and temperature non-uniformity due to uneven heating as well as thermal lag in getting to thermal equilibrium. These has been addressed elsewhere. We will instead focus on the distortion due to self-gravity and mounting. Below, we will describe our process in which a mirror segment is suspended to minimize its self-gravity sag, and bonding processes in which the mirror is temporarily bonded into a CTE-compatible strongback for metrology and subsequent alignment and mounting.

Since the optics on IXO is technically most challenging for the mission, two alternative means of mounting the mirrors are pursued in parallel in order to reduce risk associated with integrating the mirrors. Both of the mounting efforts are based on thermally formed ('slumped') glass mirrors produced at NASA's Goddard Space Flight Center (GSFC). The slumped glass technology is also developed partially for the upcoming hard x-ray mission NuSTAR, even though the two missions have different requirement in angular resolution. The two methods of mirror integration that are developed are being pursued separately at the Smithsonian Astrophysical Observatory (SAO) and at Goddard ${ }^{6,7}$. For reason which will be clear later on, the approach taken at Goddard is known generally as the "suspension mount"; whereas the approach taken by SAO is known as the Optical Alignment Pathfinder (OAP). For the suspension mount, to facilitate technology development of mirror mounting, the mounting procedure is further divided into two parts: a temporary or transfer mount where a mirror is to be temporarily mounted onto a strongback for the purposes of metrology, transportation and alignment; and a final permanent mount where the mirror is subsequently mounted onto its proper housing. The advantage of this approach is that alignment can be done separately from the permanent mounting and therefore is not necessarily limited by the environment or space around the module. There are more options in controlling the mirrors positions and orientations, and also for mirror metrology, for example. The OAP approach is to carry out the alignment in the housing module and affix the mirror in place with a single platform. This approach eliminates the need of a separate mount. The choice of having separate platforms for the temporary and permanent mounts in the suspension mount approach is also consistent with the overall mounting philosophy that the mirror figure is to remain as undisturbed as possible throughout the mounting process. This is in contrast to more active OAP approach that may attempt to adjust the mirror into its perfect form during the mounting process. In this paper, we will address the suspension mount development. Our goal is to align and mount the mirror in successive steps so that the optical figure of the mirror segment is preserved in every step. 


\subsection{Self-Gravity Distortion and Mirror Suspension}

The suspension mount process, as the name implies, begins with suspending, that is, hanging, the mirror. The mirror is hung with strings so that the mirror surface, in its near vertical orientation, remains in a minimally distorted form. Specially, the process proceeds in the following fashion: (1) A given thermally slumped mirror is suspended at two or more points by strings, so that the bulk of the mirror is vertical or near vertical. This orientation minimizes the force of gravity normal to the mirror surface. Finite-element models were used to guide the choice of the attachment points. (2) The suspended mirror is temporarily affixed to a strongback---a stiff structure made of the same material, or material with the compatible CTE---in a fashion such that all the forces involved in this fastening is sufficiently small. The bonding can most easily be made at the backside of the mirror. At this point of the development, we use pins floated in near-frictionless air-bearings, or nano-actuators with force-gauge attached, to achieve the small (sub-milli-newton) force. The attachment point can also be at the mirror's perimeter, where the force can be larger but the risk of buckling the mirror is also larger. (3) The bonded pair---the mirror and its strongback---now form an essentially rigid body, and can then transferred and situated for metrology, alignment and further processing. In particular, the mirror on its strongback can now be transferred to the module housing, be aligned optically to achieve proper focus. (4) The aligned mirror can then be 'transferred' to the housing by bonding it onto the module housing and releasing the temporary bonds. The strongback can therefore be removed to give room for the mirror in the next shell.

The suspension method is designed to minimize gravity distortion. Gravity sag of mirror in ground operation is roughly proportional to $g \sin (\alpha)$ where $g$ is the acceleration due to gravity, and angle $\alpha$ is that of the mirror (say, the center meridian) to vertical. If supported only at a small number of points, typical IXO mirrors will generally have too large a sag, except for small angle $\alpha$ (for instance, $1^{\circ}$ or less.). Therefore, to reduce the gravity distortion without violating other constraints such as the dimension and thickness, we are left with the following options: optimizing the strategic locations and significantly increase the numbers of the mounting points; or keeping $\alpha$ small by the orientating the mirror vertical. We had previously developed methods for supporting a mirror with a large $\sim 200$ number of soft support points, but denser supports are still preferred and large displacement of the mirror due to the soft springs are difficult to managed operationally. We turned to a vertical solution by suspending the mirror.

The simplest suspension method is a 2-string suspension, in which the mirror is simply suspended at two points at one end of the near-conical mirror segment. For a symmetric configuration, the separation of the suspension point is uniquely determined to ensure that the center of gravity is in the same vertical plane containing the suspension points. For example, for an ideal thin circular arc with radius $R$ and angular span $\Theta$, the chord length separating the suspension points is $L=2 R \sin (\Theta / 2) /(\Theta / 2)$, or $2 R \operatorname{sinc}(\Theta / 2)$. So, a cylindrical mirror will remain vertical when hung at such separation. For a realistic mirror, the suspension is similar except for a small correction due to its deviation from a perfect cylinder and a non-zero mirror thickness. It is also important to point out that the mirror is not necessarily in the most undistorted state with its central meridian vertical. Vertical force of gravity acting along the surface of the mirror will tend to 'drag' the mirror down and cause an out-of-plane reaction from the mirror. This self-gravity sag of the mirror in its tangential direction (approximately vertical in this orientation), even though very small, is not energetically favorable for the distortion to be sheared entirely "in-plane". The mirror responds to this gravity sag by "curing up" to create an azimuthally varying tilt, corresponding to a focus error in the focal plane. Similar "cone angle variation" along the mirror azimuth occurs regardless of whether the mirror is one in the primary or secondary stage with different cone angles, or for that matter, whether it is a cone or a cylinder. It also does not depend on the detailed of intrinsic axial curvature. For example, for a mirror with a radius of $R=242 \mathrm{~mm}, \Theta=50^{\circ}$, the variation of angular tilt corresponds to a focusing error of about 8 " rms diameter. However, unlike the sag error, the same suspension error occurring on the primary mirror and the secondary mirror, and will cancel each other. Second (axial sag) or higher order error from the two-string suspension is negligible. Distortion of a hung mirror can further be reduced by suspending the mirror at more than two points. For example, a 4-string configuration can be used.

In practice, kelvar strings are attached to the top end of the mirror, and the strings run vertically. For the dimension of mirrors under studied, sufficiently precise $L$ can be obtained in practice to assure that mirror tilt is better than, for example, $0.1^{\circ}$. Precision of $L$ necessarily depends on the size and cone angle of the mirror segment. Finite element modeling shows that mirror distortion is negligible for such small angular tilt. For the 4-string version, a whiffle-tree style implementation, with crossbars for each pair of strings, is used to reduce mirror distortion by re-distributing the load to more azimuthal positions at finer spatial scale. (A simple direct 4-string suspension is statically indeterminate, and fine adjustment of strings tensions, or lengths, especially of stiff strings, is needed.) In the whiffle-tree implementation, the tension of the strings are self adjusting (to one-quarter of the weight of the mirror). Like its 2 -string 
version, the positions of string attachment are necessarily constrained and optimized in order to have the center of gravity properly placed. To achieve negligible distortion (sub-arc-second), 4-strings suspension is needed for mirrors with $50^{\circ}$ angular span; and 2 -string suspension is sufficient for $30^{\circ}$ mirrors.

\subsection{Temporary Transfer Mount}

Having the mirror set up in suspension and thus remaining in acceptable undistorted form, the mirror is ready to be 'temporary-mounted'. To develop mounting methods, we fabricated thermally-compatible (with matching coefficient of thermal expansion, CTE) strongback for mounting mirrors. They can be fabricated from the same glass of the mirror substrate, or from metal such as titanium (Ti) or kovar. Glass strongback are exactly CTE-matched to the mirror. The CTE of Ti alloy that is reasonably close to that of the glass of the mirror, Schott D263, to within $1.5 \times 10^{-6} /{ }^{\circ} \mathrm{C}$. The CTE of the kovar material was tested to be 10 times closer, to within $0.15 \times 10^{-6} /{ }^{\circ} \mathrm{C}$ of that of the glass. Nevertheless, machining of the metal strongback can be much easier, be done more precisely and the structure can be made into much more sophisticated form. The mirror is primarily captured onto the strongback at the back of the mirror. The strongbacks are curved (or flat) structures having a number of pins for a mirror to be bonded to their tips. In order to capture the figure of the suspended mirror without introducing additional distortion, the pin can only impart a small force to the mirror. From finite element analysis, for 4-pin mounting at general position not too close to mirror's corner, force normal to the mirror surface is limited to $<1 \mathrm{mN}$ in order not to distort the mirror in its sag. The force to alter a mirror's local tilt angle is even smaller for a 4-pin mount.

Within this general context, the heart of the matter is therefore the method of capturing the mirror onto the strongback without causing unacceptable distortion. This capturing is most easily accomplished with some sort of bonding. To guide technology development, we lay out a set of requirement for this crucial process: 1) The figure of the mirror must not be degraded (by more than 0.5 arc-second, assuming similar error budget for the subsequent transfer). 2) The mirror must be bonded to the strongback with sufficient strength and in sufficient number of bonds, to allow positioning and orienting of the segment without unacceptable self-gravity distortion ( $<<0.5$ arc-seconds). 3) The bond must be debondable for transfer to a permanent housing without introducing additional displacement. 4) The temporary-bonded mirror must be sufficiently free of stress such that, after the transfer and the temporary bonds removed, the mirror figure does not change by more than 0.5 arc-seconds. 5) The locations of the bonds and associated fixtures must be compatible with subsequent alignment and permanent bonding processes. 6) The number of bond locations is to be optimized, to obtain the smallest number of points practically feasible. 7) The temporary bonding process of a mirror segment must not take more than 4 hours to complete. This last requirement, strictly speaking, is a requirement on the mounting of the IXO mirrors in the production phase, rather than in the technology development phase. Automation in the production line can improve assembly efficiency. We nevertheless include this requirement to anticipate a process that can be automated in a straightforward way.

\section{MIRROR TEMPORARY TRANSFER BOND}

\subsection{General Considerations}

Recalling that the final configuration of a module is that of a wedge of a ring, we choose to load the segmented mirrors from the back of the wedge, where the opening is largest. The module housing is therefore designed with a back end opening. With this geometry, the most straightforward way to temporarily hold the mirror for transfer is to hold it from its back. In this way, the smallest mirror of a module has to be loaded first, followed by successively larger mirrors. We have been experimenting with backside bonding and analyzing its mechanics for this configuration. The key, as stated above, is to maintain a very low force on the mirror or, equivalently, to demand that only sufficiently small displacements occur at the bond. It is easy to show that the mirror is most sensitive to small force in the direction normal to the mirror's surface, and that has been the focus of investigation.

Several geometries and mechanisms were investigated before: (1) Direct bonding of mirrors with epoxy onto tips of adjustment screws with fine pitch ( $25 \mu \mathrm{m}$ thread was used), where the screw lengths are pre-set to match the mirror at its back; (2) Bonding onto pins in low friction bearings. (3) Bonding of a mirror first to pins that sit on air-bearings. These pins have equilibrium positions pre-set to those approximately match the surface. Air-bearings have sufficiently low 'spring constant' and the normal forces acting on the mirror is determined to be sufficiently low. The pins are to be subsequently bonded to its housing. (4) Bonding of mirrors to pins that are attached to micro-force gauges and actuators. 
In this way, the forces (as small as $0.1 \mathrm{mN}$ ) can be gauged properly and can provide quantitative correlation with the result.

In addition, even in this configuration that the strongback has to be situated at the backside of the mirror, the bond points need not be limited to the mirror's back surface. Bonding locations can be, for example, at the perimeter of the mirror. The mirror can support much larger force tangentially without distorting the mirror significantly (ten's of, or up to 100 , $\mathrm{mN}$ in the mirror's axial direction, versus $<1 \mathrm{mN}$ in the surface's normal direction). On the other hand, even a small axial displacement, as small as $\sim 10 \mathrm{~nm}$ ) of mirror can cause buckling, which translates directly to sag error. We have tested mirror bonded in this way at the edge, to flexure that can flex in the axial direction. We will discuss each of these approaches in the following sections. Some of the results that was reported before are summarized.

The first method of bonding, direct bonding of a mirror onto pins on a strongback, is straightforward. It have achieved good repeatability for 4-pin mounts. In this simple scheme, a mirror in suspension is brought to contact with the pins on the strongback to within $\sim 0.2 \mathrm{~mm}$. The mirror and the strongback are then separated before epoxy beads, are applied at the tips of the pins and the mirror is brought back into contact with the pin again. However, extending this procedure to more pins in order to facilitate the transfer to an external structure encounters some difficulty. The direct insertion of a bead of epoxy to bond the mirror to the pins causes displacement of the mirror relative to the pins, and it shows up as a variation in axial tilt angle---a simple twist of the mirror---in a simple rectangular style 4-pin bond and higher order figure error in a configuration with more than 4 pins. The induced displacement is caused by a combination of the nonuniform size of the epoxy beads, uneven epoxy shrinkage and surface tension of the adhesive.

To reduce the impact of epoxy, the method is modified to get around this undesirable "epoxy-volume" effect by first bonding the mirror onto pins that are "free", so that the any change in the bond line can be accommodated. The pin is subsequently fastened onto the strongback in any mechanism in which the force is primarily directed radially on the shafts of the pins, thereby without affecting the pin in the direction normal to the mirror surface. For the choice of pins of suitable sizes, given a typical coefficient of friction of 0.5 , say, between aluminum and steel, a pin having a mass of $0.2 \mathrm{~g}$ and with a diameter of about $1 \mathrm{~mm}$ is required in order for it not to exert a force normal to the mirror surface just from frictional force. It turns out that a pin this small is not strong enough to hold the mirror in place. The bending of the pins after the removal of the suspending strings imparts bending moments local to the bond points. These local moments from pin-bending were demonstrated in surface metrology of mirrors bonded this way and the mechanical response was verified in models. Stronger pins sliding on ball bearing, thus having smaller coefficient of friction, is another option. We tested a set of linear bearing with pins of $2.7 \mathrm{~g}$ in mass and $3 \mathrm{~mm}$ in diameter. The coefficient of friction was smaller and measured at 0.12 , but because of the larger mass, the frictional force is still appreciable, at about $4 \mathrm{mN}$ normal to the mirror surface.

\subsection{Bonds to pins on air-bearings}

A set of air bearings provides very little friction to bonding pins. These work with larger pins with $6.35 \mathrm{~mm}$ in diameter. The pins are floated in their housings with sheaths of air cushion in tight gaps of approximately $10 \mu \mathrm{m}$. The "friction", measured from the angle that the pins begin to slip in either direction from its neutral position, is small $\sim 0.002$, if it is at all measurable. However, imbalanced air-flow, from imperfection in machining the tight gap where air flows, causes the pins to move forward or backward simply by air-dragging. By lightly polishing the pins, we were able to established equilibrium states of the pins so that their neutral positions are close $(\sim 1 \mathrm{~mm})$ to what are required to match the mirror surface. The pins then will gently oscillate about their equilibrium positions. Precise setting of the equilibrium positions and gentle profiling of the pins can ensure very small force from the pins onto the mirror. The restoring force is estimated, from the periods of oscillation, to be $0.1-0.4 \mathrm{mN} / \mathrm{mm}$. More direct measurement with force gauge coupled to pins on air bearings demonstrated forces as small as $0.016 \mathrm{mN} / \mathrm{mm}$ over nearly the entire length of the pin and is essentially linear. Some pins are not as good due to difference in machining. Nevertheless, such force is sufficiently small for our application.

Mirrors were bonded to the pins floated in these low friction bearings. The procedure was repeated many times, from which very good repeatability and small distortion of low order figure was achieved. The second order error is shown to repeat particularly well. The fastening mechanism of the pin to the strongback was a major source of error, as it did not entirely live up to the expectation that the adhesive only act radially onto the shafts of the pins. Surface tension of the epoxy fluid and its change during the curing phase may have contributed to the mirror's displacement. Difficulty in the application of the minute amount of epoxy also posed issues in the operation. Despite the fact that the bonding was done in an enclosure for reduce air turbulence, the mirror is nearly free-floating in this hanging mode, with the pins attached. 
One of the more unexpected issue is the cleaning of the air-bearing after each bonding. It was difficult to clean these pins and bearings and to restore them to the original state perfectly well. So, despite early success, the bonds occasionally were not as good as expected. The process can further be developed, by improving the operation (quieter environment for operation, better cleaning methods).

Figure 1. Mirror bonded on pins which were seated in air-bearings. The pins are modified so that they, when placed horizontally, were able to stay in their equilibrium positions that were designed to match the geometry of the mirror. The oscillations of the pins about their equilibrium positions are sufficiently gentle that the force along the shafts, and thus normal to the surface of the mirror, were small for reasonable amplitude of displacement. (Left) A mirror bonded to the tip of the pins. (Right) The backside of the strongback, showing the manifold that supplied air to float the pin. The pins are subsequently bonded to caps on the bearings' housings.
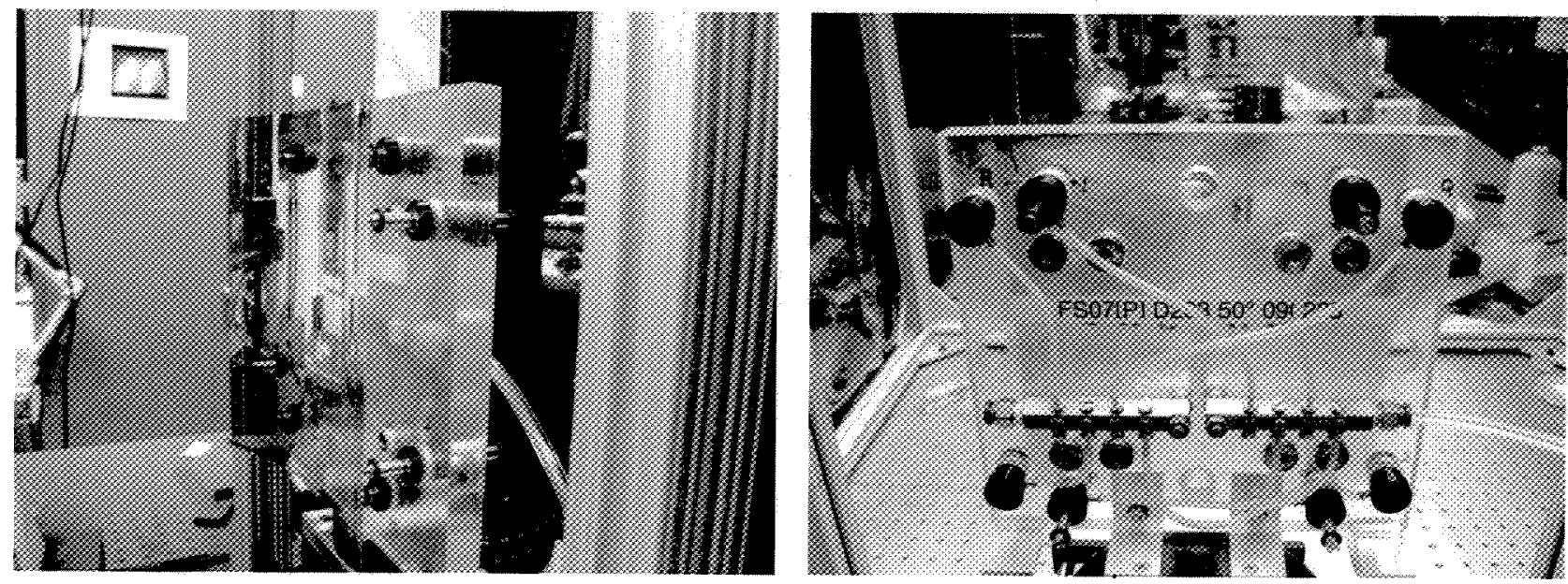

Figure 2. Low order parameters characterizing a mirror bonded to pins with air-bearings, as a function of the mirror's azimuth. The parameters were derived from interferometric measurement of the mirror surface. The top panel shows the variations of overall radius. The middle panel shows the variation of the cone angle across azimuth. The bottom panel shows the axial sag.

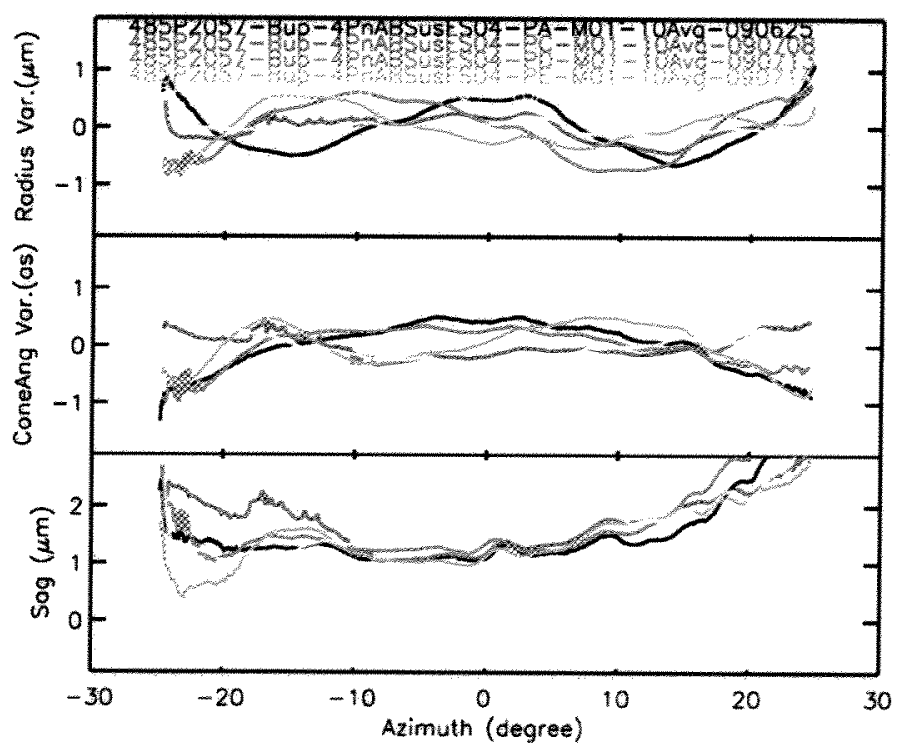

Figure 2 shows the repeatability in a series of measurement of a mirror bonded to the pins on air-bearings. The mirror radius is nominally $242.5 \mathrm{~mm}$ and spans 50 degrees. Bonds are done at four points, with two in each azimuths where the mirror was first suspended. Low order repeatability is in general quite good. Dependence on azimuthal angle of radius variation is unimportant at this level, as the image is insensitive to radius change at a few micrometers. We noted that the absolute radius and a linear dependence could not be obtained from the measurement due to the nature of interferometry and the orientation (roll angle) of the cylindrical null. The tilt angle ("cone angle") variation at arcsecond level is important as it focus the image at particular azimuthal subaperture at different position in the focal plane. Again, we noted that the result contained a component of cone angle variation that could not be separated from a simple tilt of the cylindrical null. 


\subsection{Bonds to pins with control force}

The methods described above relied on qualifying the (small) force, before hand, that the mount will have on the mirror. This was done by modeling as well as measurement with a separate set up. A better way is to have direct knowledge of the force in real time. To more quantitatively control the force of the pin to which the mirror is bonded, a micro-force gauge was attached to each pin and was driven at the back with an actuator. The gauges are capable of measuring forces along the pin/shaft direction with a resolution better than $0.01 \mathrm{mN}$. The actuators are capable of driving the shaft with a resolution of $10 \mathrm{~nm}$. These are adequate precisions for the present purpose. In the present configuration, a mirror is suspended from the 4-string suspension mount described above, and therefore free from any significant distortion due to gravity. The whole platform is placed in front of a interferometer with a set transmission cylindrical null lens. The set of cylindrical null lens converts the planar wave front from the interferometer to a cylindrical wave front, allowing measurement of the mirror in a single shot. The interferometer, employing a dynamical interferometry, permits measurement of the hung, and therefore mildly swinging, mirror. On the mounting platform, three of the pins, in a triangular configuration with one near the top and two near the bottom, were used to slightly adjust the orientation of the mirror in such a way that the it was measurable with the interferometer, and the mirror was pushed into position with known and acceptable forces. The actuators are controlled automatically with close-loop feedback from the force sensors with defined forces. Because of the automatic adjustment of the pins' forces, this mounting platform, containing a strongback (currently made of kovar), pins, actuating mechanisms, force gauges and controllers, is referred to as the 'smart pin' metrology/mounting platform.

Figure 3. Smart-pin experimental platform. (Left) The picture shows the backside of the platform and the instrumentation. Three actuators were used for stabilizing the mirror before bonding and for adjusting the mirror with sufficiently low force. A set of displacement sensors mounted separately were used to acquire neutral positions of the mirror. Four other actuators were used to maintain the bonded mirror continuously to zero-displacement. (Right) A force gauge at the top of the fixture was used to maintain low forces.
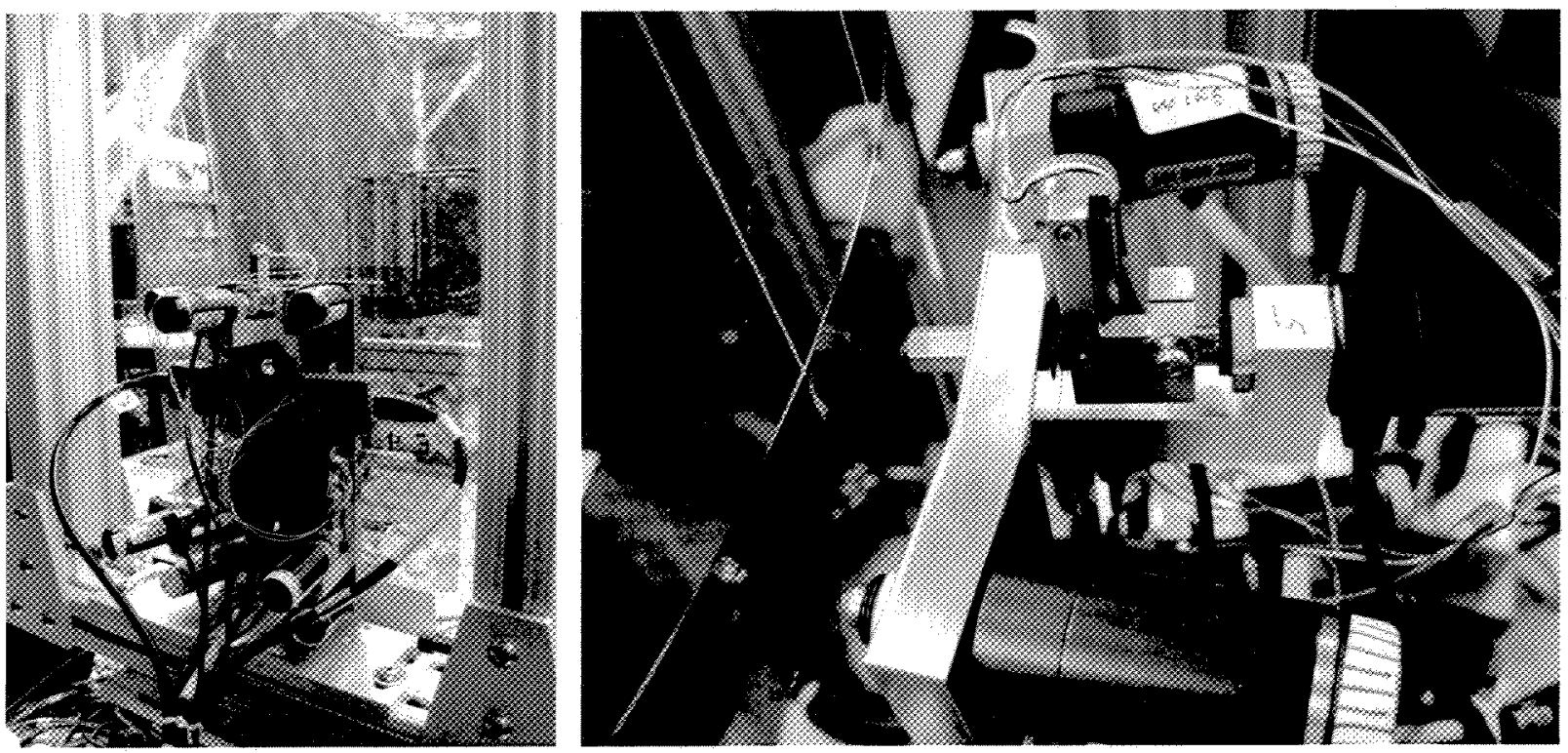

A series of measurements were carried out to test the repeatability of the forces and also of the measured parameters. In the test, a mirror was hung with a 4-string suspension mount, stabilized by the three actuated pins. The mirror was originally not exactly vertical, by design, and it only leaned on the three actuated pins. The simple contact between the pins and the mirror allowed, barring negligible frictional force, complete freedom of the mirror in the direction tangential to the mirror surface. The stabilization is accomplished with close-loop control with a set of input force parameters. The input forces were pre-determined from a preliminary test. For the dimension of the mirrors and the suspension parameters we used, the forces were typically $1 \mathrm{mN}$ at the bottom pins and $0.1 \mathrm{mN}$ at the top pin. The same mirror was measured five times in the repeatability test. It was found that the repeatability is excellent with these smart pins. Figure 4 shows the result, broken down in low order axial terms, as a function of azimuths. 
The metrology mount was extended to perform also as a mounting platform by simply adding more actuating pins. A rectangular configuration of 4 pins was chosen. In this conception, the mirror are stabilized with the three pins as described above. The mounting four pins are then zeroed to provide reference to zero force. The mirror is subsequently bonded onto the four mounting pin and zero force will be maintained then with the same close-loop control. In practice, UV-cured epoxy will be used with the epoxy pre-deposited on the pins' heads. A technique of curing the epoxy with UV radiation in multiple steps was also developed for this and other purpose. The fabrication of hardware and building of platform was recently completed. Preliminary runs of tests to check out the functionality of the system were in progress. A series of tests of 'zero-force' bonding is planned but, as this manuscript goes to the publisher, the tests on actually bonding a mirror have not yet been carried out.

A set of displacement sensors were mounted separately to monitor specific position of the mirror to be bonded. The sensors were mounted separately to avoid disturbance to the mirror mount from the sensors and its controllers. Neutral displacement levels were obtained from long term averaging of the mirror position before bonding, as the mirror suspended was not completely steady. Oscillations with an amplitude of about $10 \mu \mathrm{m}$ could be achieved in the experimental environment where the mirror is placed in an enclosure. Sensor data were used as feedback for a close-loop actuation in the bonding process.

Figure 4. Smart-pin metrology. Low order parameters from a repeatability test of the smart-pin platform, used as a metrology mount. The mirror was suspended but not bonded. Excellent repeatability were obtained in different runs where forces to stabilize the mirror was set to obtain surface measurement with an interferometer.
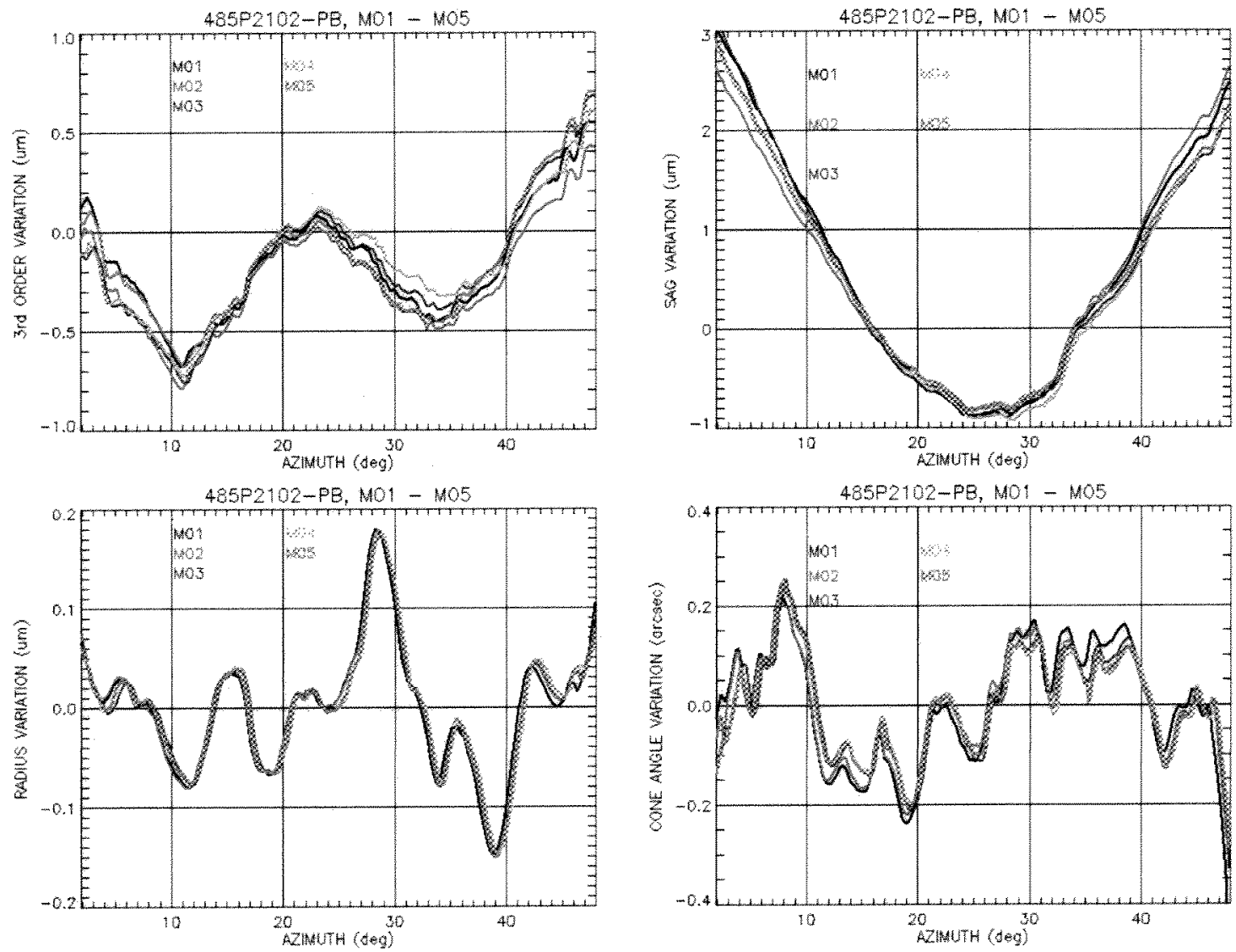

\subsection{Edge bonding}

In contrast to the bonding onto the mirror's backside, bonding to the mirror's thin edge offers some advantage. If the bond is properly placed at the thin edge, most of the force will be directed along the mirror's surface, rather than perpendicular to it, which is more detrimental. If bonded to the mirror edge at the axial ends, the mirror is also much stiffer and therefore can support much greater forces. Modeling shows that for a mirror bonded at six axial locations (three each at the mirror's top and bottom), the mirror can support ten's of $\mathrm{mN}$ before the mirror buckles and shows 
significant axial sags. The force limit certainly depends on the size of the mirror and the positions of the bonds. The geometry may not be the most straightforward to be incorporated into the module housing described above, nor it is easy to apply a small bead of adhesive within the thickness $(0.4 \mathrm{~mm})$ of the glass segment, but these certainly are not limits in a fundamental sense.

Figure 5. (Left) Mirror was to be bonded to a rigid pin at the bottom. The pin is in the form of a screw that can be coarsely adjusted. The set up is placed on a precision vertical stage that is used to adjust the precise vertical position of the mirror relative to the fixed pins. Epoxy was not applied yet. (Right) Mirror is bonded at the top to thin slivers. The slivers are sufficiently soft to absorb any dimension adjustment in the vertical/axial direction but yet sufficient stiff to hold the mirror in place in the horizontal plane.
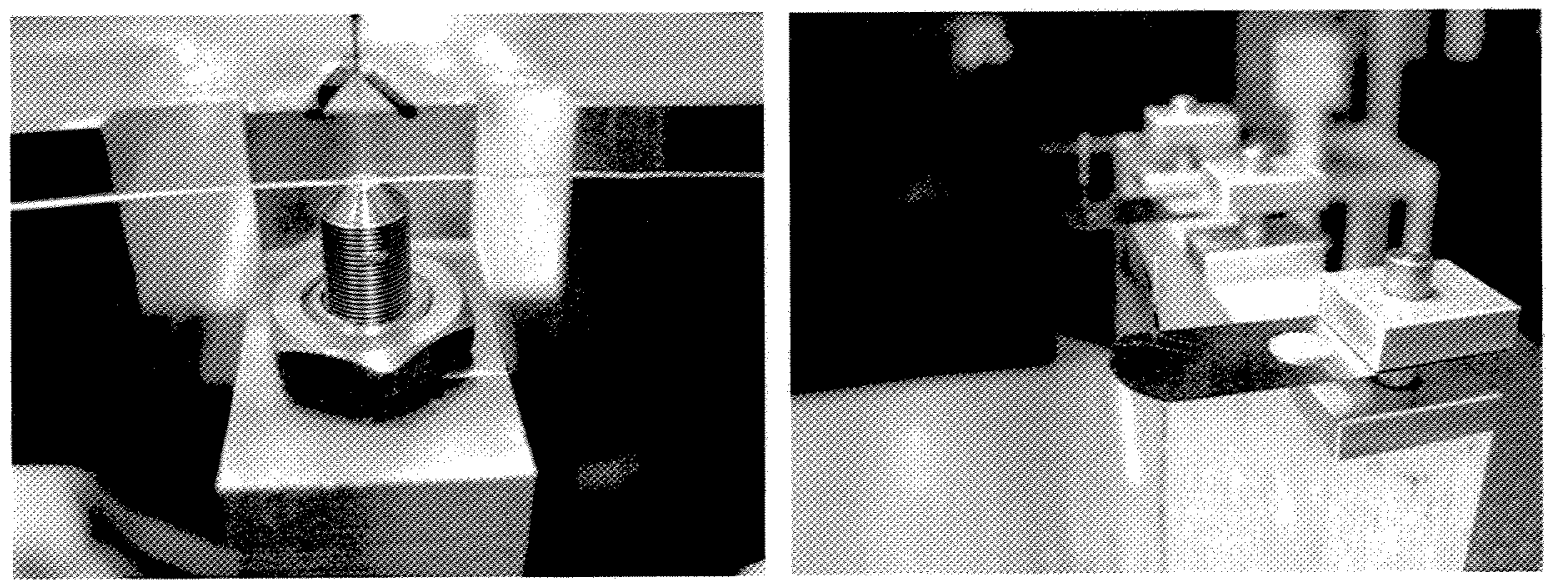

Figure 6. Low order figure variations from edge-bond mount. The top panel shows the radius variation. As noted before, the absolute radius could not be obtained from such measurement. The middle two panels show the cone angle and sag variations, which were very uniform. The bottom panel shows the residual surface after these three low order terms were removed.

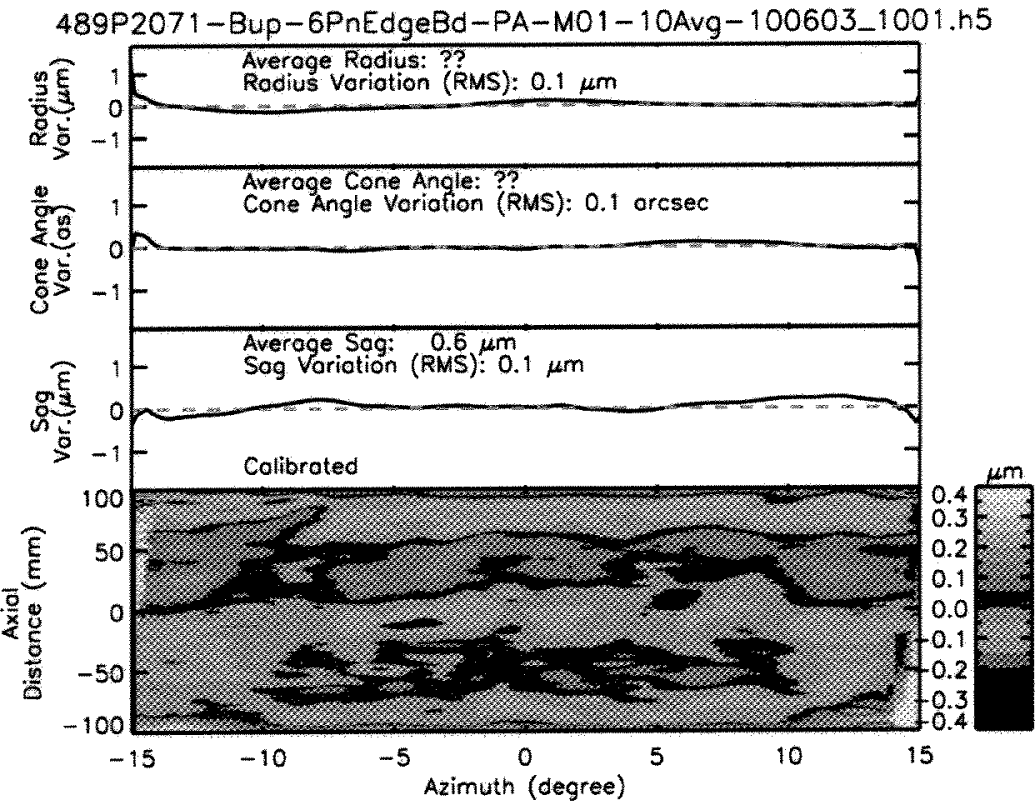

We have tested this concept as an alternative to the default backside practice. It was found that, to achieve the still rather small force that is required, the pin (what we generically call the structure that links the mirror to the strongback) has to be rather small/thin. If the pins align more or less with the plane of the mirror's local surface, that is, the pin being vertical, there are severe restrictions on the weights of the pins. Moreover, it is not easy to position the pins in this orientation. Based on these considerations, we settled on the following geometry that is placed horizontally instead: a set up in which rigid pins were positioned at the bottom, with height adjustment to meet the mirror edge, and a set of thin flexures on the top. Figure 5 shows the joints of the mirror to the strongback. The flexure is essentially a thin sliver in that they can flex in the vertical direction but can support the mirror well in the horizontal plane. To satisfy the small force requirement, we used $75 \mu \mathrm{m}$ thick, $5 \mathrm{~mm}$ wide, and $1 \mathrm{~cm}$ long slivers. Such thickness can readily be available in the form of stainless steel shims. The 
thermal compatibility of the stainless steel material is certainly far from ideal, but it was useful for a proof of concept. We also experimented with CTE-matched glass slivers, even though they are not quite available in such thickness. The slivers used had stiffness of $\sim 0.1 \mathrm{mN} / \mu \mathrm{m}$, which is quite adequate for the purpose as a flexure.

Preliminary result was encouraging. We were able to obtain result with very small low order errors in the first few trials. Figure 6 shows an early attempts demonstrating $\sim 0.1 / \mu \mathrm{m}$ of sag $(\mathrm{rms})$ variation. This was expected since the flexures were designed to have low axial forces. What is more interesting is that the bonding consistently shows a very uniform first order ("cone angle") variation across the azimuth. This is more surprising since the sized of the epoxy beads used was not really as fine as the thickness of the mirror, implying possible imbalance of force from the asymmetric distribution of the epoxy at the front and back of the mirror. The exact nature of such cone angle variation is being investigated with displacement sensors.

\section{ALIGNMENT AND PERMANENT MOUNTING INTO HOUSING}

The mirror bonded with one of the temporary/transfer mount described above was aligned and subsequently transferred from the temporary mount to a permanent structure. The process is currently studied with the aid of a Mirror Housing Simulator ${ }^{8}$. This simulator is a framed structure made of titanium, Ti-15Mo, and consists of bonding tabs, also made of titanium, on short rails at its sides (and top and bottom ends.) The mirror housing simulator can accommodate the three pairs of mirrors of different radii which are currently available. It is kinematically mounted in the vertical orientation on its base plate. Mirror to be bonded onto the housing simulator is transferred from the strongback where the mirror is temporary-bonded. Mirror bonded on the strongback in the temporary mount can be oriented and aligned with the aid of a 6 degree-of-freedom hexapod. The mirror was subsequently bonded to the tabs of the housing simulator. The bonding process employed a set of displacement sensors to monitor the displacement of the mirror due to bonding (forces due to the application of epoxy, epoxy's surface tension, and curing). Epxoy was injected from a syringe which is mounted on an actuator. An UV-cured epoxy was used. The position data were fed back to the actuator to maintain good displacement. The mirror was subsequently de-bonded from the strongback. The de-bonding process was tested repeatedly in test fixtures and was shown to be reliable. Detailed discussion of the alignment and permanent bonding processes are reported in a separate publication in this conference.

Figure 7. The mirror alignment and permanent bonding onto housing simulator. (Left) The housing simulator with associated instrumentation. (Right) Mirrors mounted on the housing simulator.
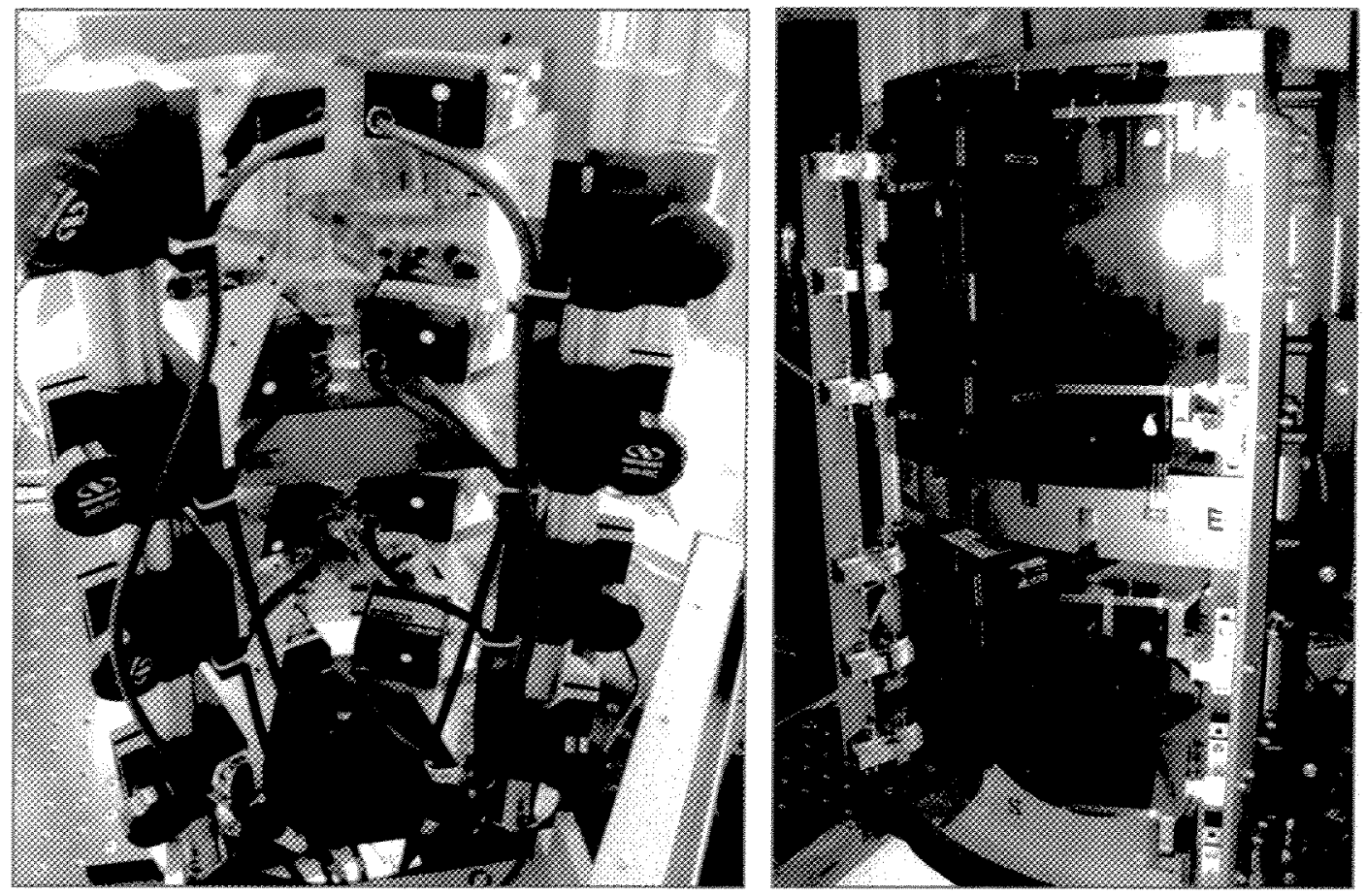


\section{SUMMARY}

In summary, we have developed a general scheme of mounting method for the segmented mirrors for the IXO mission. The approach aims at mounting the mirrors without introducing distortions, as opposed to any attempt to adjust the performance along the way. The mount starts with suspending the mirror for negligible distortion, establishing a baseline for subsequent bonding. Metrological methods were also developed to characterize the mirror at this state. Several options of temporary/transfer mounting are still open, and optimization is being made. The down selection of a prefer temporary mount will be made towards the end of this year. Progress in alignment of mirrors and permanent bonding onto the housing simulator were made and is expected to achieve sufficient accuracy. A comprehensive series of tests are planned, which will include optical metrology of the mirror's performance in various stages, and final x-ray tests.

\section{REFERENCES}

[1] Bookbinder, J., "The International X-Ray Observatory: Activity submission in response to the Astro2010 Program Prioritization Panel RFI," Internal and submitted document in response to the Astro2010 Decadal Survey (2009).

[2] David W. Robinson and Ryan S. McClelland, "Mechanical Overview of the International X-Ray Observatory", in IEEE Aerospace Conference 2009, AERO.2009.4839581 (2009).

[3] Ryan S. McClelland and David W. Robinson, "Design Concept for the International X-Ray Observatory Flight Mirror Assembly", AERO.2010.4839431 (2010).

[4] W.W.Zhang, et al. "Mirror technology development for the International X-ray Observatory mission", Proc. SPIE 7437, 74370Q (2009).

[5] Kotska Wallace, Marcos Bavdaz, Philippe Gondoin, et al, "Silicon pore optics development", Proc. SPIE 7437, 74370T (2009)

[6] Kai-Wing Chan, William Zhang, Timo Saha, et al., "An approach for alignment, mounting, and integration of IXO mirror segments", Proc. SPIE 7437, 74371D (2009).

[7] Mark D. Freeman, Paul B. Reid, William Podgorski, and David Caldwell, "Progress on the active alignment system for the IXO mirrors", Proc. SPIE 7437, 74371E (2009).

[8] Tyler C. Evans, Kai-Wing Chan and Timo Saha, "Arc-Second Alignment of International X-Ray Observatory Mirror Segments in a Fixed Structure", in IEEE Aerospace Conference 2010, AERO.2010.5446900 (2010). 